\title{
Some aspects of particle acceleration and transport at CME-driven shocks
}

\author{
G. Li and G. P. Zank ${ }^{1}$ \\ ${ }^{1}$ IGPP, University of California at Riverside, CA 92026, USA \\ email: gang.li@ucr.edu, zank@ucr.edu
}

\begin{abstract}
Gradual solar energetic particle (SEP) events are now believed to be associated with CME-driven shocks. As the shock propagates out from the Sun, particles are accelerated diffusively at the shock front and some will escape upstream and downstream into the interplanetary medium. This is in contrast with "impulsive" events, which are believed to be due to solar flares. However, recent observations have found that in some gradual SEP events, the time intensity profile show a two peak feature, suggesting a mixture of particles from solar flares with particles from CME-driven shock. Furthermore, the observed spectra of large SEP events show tremendous variability. The $\mathrm{Fe} / \mathrm{C}(\mathrm{Fe} / \mathrm{O})$ ratio behave oppositely in events which have similar solar progenitors. In this work, we use a numerical model to follow particle acceleration and transport at CME-driven shocks. We investigate a possible scenario for the re-acceleration of flare particles by CME-driven shocks and calculate the $\mathrm{Fe} / \mathrm{O}$ ratio for two exemple shocks. These simulations are helpful in interpreting observations of particle data obtained in situ at 1 AU by spacecraft such as ACE and WIND.
\end{abstract}

Keywords. shock waves, Sun: coronal mass ejections (CMEs), particle emission

\section{Introduction}

Solar flares and Coronal Mass Ejections (CMEs) are catastrophic solar events. Energies up to $10^{31-32}$ erg are released in typical CMEs and large flares. In both cases, particles can be accelerated up to $\sim \mathrm{GeV} / \mathrm{Nuc}$. The underlying mechanism of each process, however, is still presently unknown, although magnetic reconnection is widely believed to be the cause of both phenomena. Observationally, solar flares usually happen in active regions and are not spatially extended. CMEs, on the other hand are spatially extended and have an average angular width of 50 degree (see for example Burkepile (2004)). Furthermore, large CMEs are likely to have flares associated with them (Kahler (1984) and Cliver et al. (2004)).

Evidence of electron acceleration in both cases can be inferred from the accompanying X-rays (Johns \& Lin (1992)), which result from the interaction of accelerated electrons and ambient solar atmosphere. One important question for understanding the x-ray spectrum is how much X-ray emission is due to non-thermal electrons. Since non-thermal electrons lose their energy mainly through Coulomb scattering and X-rays only amount up to $10^{-5}$ of the total released energy, thus an X-ray due to non-thermal electrons would impose a strong relationship between the energy release process and the particle acceleration process (Lin (2002)). Similarly, the acceleration of ions can be inferred from various nuclear Gamma-ray lines. These nuclear Gamma-ray lines are produced when energetic ions collide with ambient protons and/or alpha particles in the solar atmosphere (corresponding to broad gamma-ray lines) or when energetic protons collide with ambient heavy ions (corresponding to narrow gamma-ray lines). 
Besides various observations aimed directly at the solar surface, in-situ observations of Solar Energetic Particles (SEPs) provide another way for studying CMEs and flares. Historically, SEP events have been classified into two categories, "impulsive" and "gradual". The nomenclature was used initially to refer to the duration of the associated soft X-ray signals, and later on was refined as a classification of "flare accelerated" (the "impulsive") and CME-driven shock accelerated (the "gradual") events (see for example, Reames 1997 and Cliver and Cane (2002)).

Earlier studies of energetic particle abundances (see for example, Mason et al. (1984)) have found that for "impulsive" flare events, the measured states of Si and Fe indicate a hot temperature of $\approx 2 \times 10^{7} \mathrm{~K}$, close to that of the hot flare plasma. On the other hand, the ionic charge states for "gradual" CME-driven shock events tend to correspond to a temperature of $\approx 2 \times 10^{6} \mathrm{~K}$, suggesting a source of a cooler coronal material (Mason et al. (1995) and Tylka et al. (1995)). However, as more data were obtained, especially after the launch of spacecraft ACE in the past decade, one sometimes finds examples of SEP events which possess impulsive characteristics but are associated with CME events. Recently, Cane et al. (2003), studied 29 intense SEP events and found four mixed events. These events have a time intensity profile that looks like those due to CME-driven shock acceleration, but have a flare-like composition. It is possible that these are events in which CMEs and accompanying flares occur temporally close to each other and some of the solar flare material will undergo shock re-acceleration (see Li and Zank (2004a)), thus having a time intensity profile similar to shock acceleration particles but having a flare-like composition.

Another noteworthy phenomenon in large SEP events is the spectral variability. In a recent survey, Tylka et al. (2004) found that about $1 / 3$ of the large SEP events observed by ACE and Wind in Solar Cycle 23 exhibit explicit energy dependence in $\mathrm{Fe} / \mathrm{C}$. However, the energy dependence of $F e / C$ vary significantly between events that have similar solar progenitors. For example, the CME ejections of two large SEP events, 21 April 2002 event and the 24 August 2002 event, are very similar, $\dagger$ but the $F e / C$ ratio above $10 \mathrm{MeV} /$ Nuc.behaves very differently. The $\mathrm{Fe} / \mathrm{C}$ ratio decreases with energy for the 21 April 2002 and increases with energy for the 24 August 2002 event. Tylka et al. (2004) has invoked particle acceleration at a perpendicular shock to explain the opposite energy dependence of $\mathrm{Fe} / \mathrm{C}$ in these two events. They argued that the 24 August 2002 event may correspond to a perpendicular shock, which has higher injection energies. Since more remnant flare material are populated at higher energies, and there are relatively more seed $\mathrm{Fe}$ ions than seed $\mathrm{CNO}$ ions, we thus expect to see a different energy dependence of $\mathrm{Fe} / \mathrm{C}$ in the 24 August 2002 event from that of the 21 April 2002 event.

In this paper, we use the model developed initially by Zank et al. (2000) and later improved by Rice et al. (2003) and Li et al. (2003) to follow the acceleration and transport of protons and heavy ions at a CME-driven shock. In the following, we discuss the possible re-acceleration of flare particles and perform a simulation that calculates the $\mathrm{Fe} / \mathrm{O}$ ratio at two exemple shocks. For details of the model, readers are refereed to the above mentioned papers.

\section{Re-acceleration of flare particles}

Large CMEs are usually associated with solar flares, and to understand the corresponding SEP observations, it is crucial to understand the temporal relationship between CMEs

$\dagger$ The initial CME speed and transit time to Earth are $2400 \mathrm{~km} / \mathrm{s}, 51$ hours and $1900 \mathrm{~km} / \mathrm{s}$, 58 hours for the 21 April 2002 and the 24 August 2002 events respectively. The associated solar flares for these two events are also comparable with the 21 April 2002 event being X1.5/1F at $S 14^{\circ} W 84^{\circ}$ and 24 August 2002 event being $\mathrm{X} 3.1 / 1 \mathrm{~F}$ at $S 02^{\circ} W 81^{\circ}$ 
and associated solar flares. Zhang et al. (2001), using C1, C2 and C3 of LASCO and X-ray data from GOES studied four CME events and found that the main CME acceleration and the main energy release of associated flare occur almost simultaneously. If CMEs and associated solar flares occur at approximately the same time, then we would expect to see some interaction between processes associated with these two events. Li and Zank (2004a) studied the re-acceleration of flare particles by assuming particles are accelerated at both solar flares and CME-driven shocks. The spectrum of the flare particle is assumed to be $p^{-4}$ and the acceleration last 1000 seconds. The spectra of CME-driven shock particles are followed numerically. When particles escape upstream of the shock, they propagate along the interplanetary magnetic field (IMF) with occasional pitch angle scatterings. Because of the presence of the CME-driven shock, large portion of the flare particles are subject to absorption by the shock and are re-accelerated. Figure 1 is a plot of the time-intensity profile adapted from Li and Zank (2004a). In the plot, the upper panel assumes particle acceleration due to a pure flare source. The second panel assumes particle acceleration due to a pure shock source and the third panel assumes a mixed source with a ratio of the initial number of CME-driven particles to the initial number of flare particles to be $2: 1$. The particle mean free path in front of the shock due to pitch angle scattering is taken to be $\lambda=0.4 \mathrm{AU}\left(\frac{p c}{1 \mathrm{GeV}}\right)^{1 / 3}\left(\frac{r}{1 \mathrm{AU}}\right)^{2 / 3}$ in all three panels. From figure 1 we can see that the time intensity profile for the "flare-only" case (the upper panels) shows an abrupt rise followed by a decay. This is because the point source lasts a short time only. The middle panel of Figure 1 corresponds to the "shock-only" case. Compared to the "flare-only" case, it is clear that the traveling shock, which is neither spatially nor temporally confined, leads to a much slower rise of the intensity profile. The bottom panel of Figure 1 corresponds to the "flare + shock" case. At early times, the intensity profile resembles the "flare-only" case. These are particles that were accelerated at the flare site, and then propagate to $1 \mathrm{AU}$ and not absorbed by the shock. At later times, the contribution from the shock becomes more pronounced. The contribution comes from two populations, these being particles that were injected from the ambient solar wind and accelerated at the traveling shock, and particles that were accelerated at the flare but have been absorbed and re-accelerated by the shock. The latter particles, once absorbed, will behave like those particles that originate at the shock. Thus, at late times, we expect the intensity profile of the "flare + shock" case to behave like that of the "shock-only" case. Indeed, the profile shown in the bottom panel resembles that observed by Cane et al. (2003).

\section{The $\mathrm{Fe} / \mathrm{O}$ ratio}

Understanding how heavy ions are accelerated at CME-driven shocks and then transported in the interplanetary medium is essential to understand the observational data obtained by spacecraft such as ACE and WIND. Unlike protons, which comprise $\sim 98 \%$ material in the solar system and when accelerated at the CME-driven shocks, will generate Alfvén waves, heavy ions such as $C, N, O$ and $F e$ are "rare" elements and can be treated as test particles. The differences between various heavy ions are the charges and masses. Even though the diffusive shock acceleration mechanism does not differentiate between ions of different charges and masses and predicts power laws for different ions with the same spectral index, the maximum attainable energy for each element, however, depends on the combination of $Q / A$. Here $Q$ is the charge of the heavy ion and $A$ is the mass number. Furthermore, the mean free paths of ions also depend on $Q / A$. Thus the spectra observed at $1 \mathrm{AU}$ of different heavy ions can be quite different. One useful observational quantity of an SEP event is the $\mathrm{Fe} / \mathrm{O}$ ratio as a function of time at 


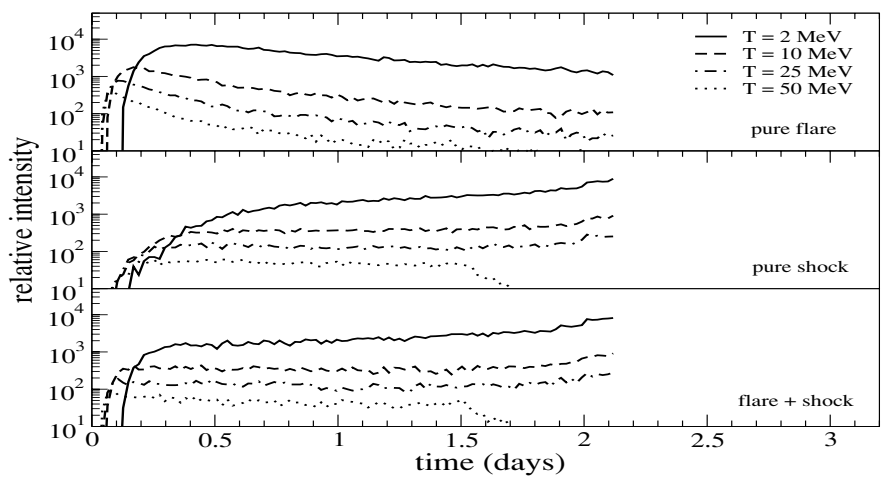

Figure 1. The re-acceleration of flare particle by a CME-driven shock. From Li and Zank (2004a). The upper panel assumes particle acceleration due to a pure flare source. The second panel assumes particle acceleration due to a pure shock source. The third panel assumes a mixed source. See text for details.
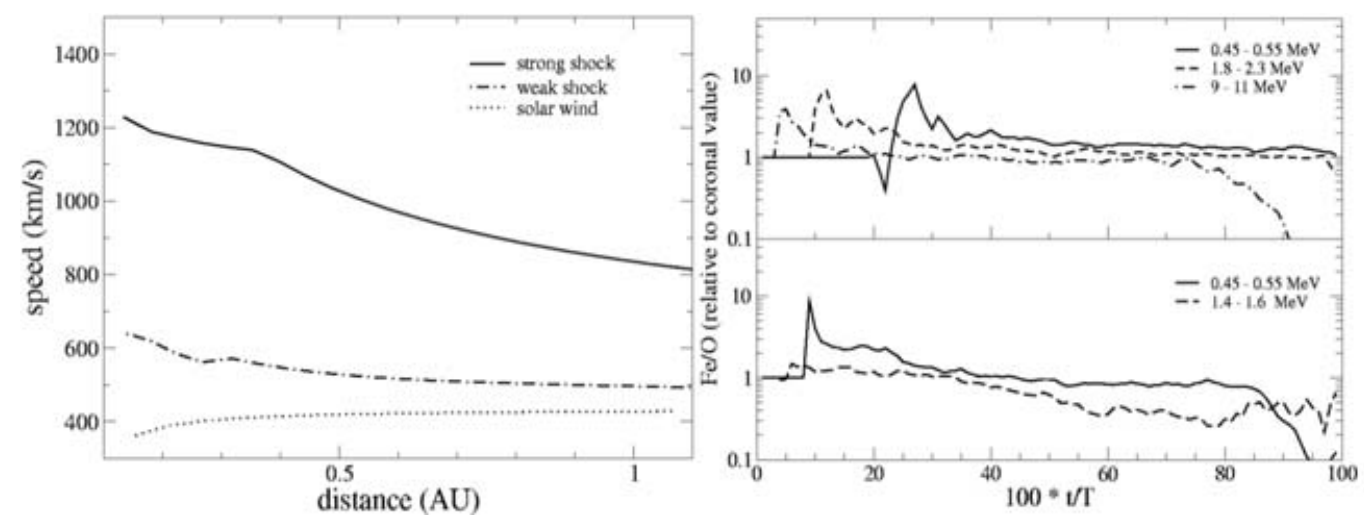

Figure 2. The shock profile for a strong and a weak shock and the corresponding $F e / O$ ratio (from Li and Zank (2004b)). The left part plot the shock speed as the function of heliospheric distance for a strong and a weak shock. The right part plot the $\mathrm{Fe} / \mathrm{O}$ ratio as a function of time.

different energies. Li and Zank (2004b) performed simulations of heavy ion acceleration and transport at a strong and a weak shock and obtained the corresponding $\mathrm{Fe} / \mathrm{O}$ ratio, shown in Figure 2. The left part of Figure 2 is the shock profile. The solid line is for the strong shock and the dashed line is for the weak shock. The solar wind speed is also plotted in the figure for reference. The right part of Figure 2 contains the $F e / O$ ratio. The upper panel is for the strong shock and the lower panel is for the weak shock. In the upper panel, three energy bins: 0.45 - $0.55 \mathrm{MeV} / \mathrm{Nuc}$, $1.8-2.3 \mathrm{MeV} / \mathrm{Nuc}$. and 9 $11 \mathrm{MeV} /$ Nuc. are shown. In the lower panel, two energy ranges, $0.45-0.55 \mathrm{MeV} / \mathrm{Nuc}$. and $1.4-1.6 \mathrm{MeV} / \mathrm{Nuc}$. are shown. From the figure we see that the $F e / C N O$ ratios rise above 1 at early times and then slowly decrease, approaching 1 or even going below 1 at later times. The curve corresponding to higher energies rises faster, but has a smaller peak value. The early rise of the $F e / C N O$ ratio is because iron, at the same energy per nucleon, has a longer mean free path than CNO particles, thus arriving at $1 \mathrm{AU}$ sooner. The ratios shown in Figure 2 are consistent with observations. (Tylka et al. (1999)). 


\section{Conclusion}

With the launch of spacecraft such as ACE, WIND and RHESSI in the past decades, much data for SEP events has been obtained. These include X-ray, gamma ray, radio signals at or near the surface of the Sun and in-situ particle and magnetic field data at 1 AU. We now know that large gradual SEP events are due to CMEs and large CMEs are often accompanied by solar flares. However, the exact relationship between CMEs and solar flares is still unknown. Equally intriguing questions are, for example, how particles are accelerated at CME-driven shocks; what is the seed population and can perpendicular shock acceleration explain the spectral variability? To answer these questions, it is essential to develop a sophisticated numerical model which captures the essence of the underlying physics. In this paper, using a model we developed earlier, we discuss the possibility of a mixture of flare accelerated particles with CME-driven shock accelerated particles and its possible implications for the time intensity profiles of SEP events. We also considered the $\mathrm{Fe} / \mathrm{O}$ ratio as a function of time for a parallel shock configuration and find qualitative agreements with observations. We believe this work is helpful on interpreting the observations of particle data obtained in situ at $1 \mathrm{AU}$ by spacecraft such as ACE and WIND.

\section{Acknowledgements}

This work has been supported in part by a NASA grant NAG5-10932 and an NSF grant ATM-0296113. GL would like to thank Drs Y.H. Yan and J. Wang for their hospitality during his stay in Beijing.

\section{References}

Burkepile, J. T. et al. , 2004, J. Geophys. Res. 109, A03103 doi: 10.1029/2003JA010149.

Cane, H.V., et al., 2003, Geophy. Res. Lett. 30, 10.1029/2002GL016580.

Cliver, E.W., Cane, H.V., 2002, EOS Vol. 83, number 7.

Cliver, E. et al. , 2004, ApJ, 605, 902-910.

Johns, C. and Lin, R. P., 1992, Sol. Phys. 137, 121.

Kahler, S.W. et al., 1984, J. Geophys. Res. 89, 9683.

Lin, R. P. et al., 2002, Sol. Phys. 210, 3.

Li, G, et al., 2003, J. Geophys. Res.,108(A2), 1082, doi:10.1029/2002JA009666.

Li, G. and Zank, G. P., 2004a, Geophys. Res. Lett., in press.

Li, G. and Zank, G. P., 2004b, J. Geophys. Res., submitted.

Mason, G.M., Gloeckler, G., Hovestadt, D., Astrophys. J., 280, 902-916.

Mason, G.M., et al., 1995, Astrophys. J., 452, 901-911.

Reames, D.V., Kahler, S.W., and Ng, C.K., 1997 Astrophys. J., 491, 414-420.

Rice, W.K.M., et al., 2003, J. Geophys. Res., 108, A10, pp. SSH 5-1, doi:10.1029/2002JA009756.

Tylka, A.J., et al., 1995, Astrophys. J., 444, L109-L113.

Tylka, A.J. et al., 1999. Geophys. Res. Lett., 26, 2141-2144

Tylka, A. et al. , 2004, submitted to ApJ.

Zank, G.P. et al., 2000, J. Geophys. Res., 105, 25079-25095.

Zhang, J., et al., 2001, Astrophys. J., 559, 452. 


\section{Discussion}

P.F. CHEn: Which parameters govern the final spectral index of the energetic particles in your model?

LI: The spectrum at the shock front is decided by the instantaneous shock parameters such as compression ratio, etc. The observed particles at $1 \mathrm{AU}$ is a combination of particle acceleration at the shock front and particle transport in the interplanetary medium. 